\title{
Les phytosanitaires - homologation
}

\author{
Plant protection products - homologation \\ par A. Rico \\ Professeur Émérite \\ École Nationale Vétérinaire de Toulouse
}

The aims of the registration of pesticides in Europe are to define their security of use for consumers, workers and environment. To achieve this goal, we have in France the necessary structures described in the first part of the paper (Comité d'homogation, Commission des Toxiques en Agriculture, structures scientifiques mixtes, etc.). Risk is function of hazard and exposure. In terms of hazard, the valid concept is to get no effect level in different targets. However, new concepts will be introduced in the future concerning carcinogenicity, hormonotoxicity and immunotoxicity. Another important problem is the toxicity of the mixtures. In terms of exposure, it is necessary to get information on the real exposure for consumers, workers and water. With the development of analytical technologies, it is a difficult exercise but now possible. However, the regulatory process must be more than anything else a scientific exercise. The presence of traces of pesticides in the environment is not necessarily a danger. Only scientific data can permit a good estimation of the risks.

\section{I $\square$ LES TEXTES ET LES STRUCTURES D'HOMOLOGATION}

L'homologation des produits phytosanitaires en France s'appuie sur des textes et des structures. En ce qui concerne les textes, le document fondamental est la Directive 91//414/CEE qui définit en particulier l'ensemble des informations scientifiques à fournir pour demander un enregistrement soit d'une matière active, soit de formulations. Cette directive a été transposée en droit français. En ce qui concerne les structures, il existe deux instances : la Commission d'Étude de la Toxicité des Produits Antiparasitaires à Usage Agricole et le Comité d'Homologation. Le Comité d'Homologation propose au ministre des décisions compte tenu de l'avis de la Commission des Toxiques. Cette dernière, qui vient d'être renouvelée, comprend des toxicologues dont l'objectif est d'estimer les risques d'utilisation des produits pour le consommateur au travers des résidus, le manipulateur et l'environnement. Pour réaliser sa mission, elle est confortée par une structure récente : la structure scientifique mixte, comprenant des chercheurs de l'I.N.R.A. et de la D.G.A.L., travaillant à temps plein et assurant aussi le secrétariat scientifique. Le secrétariat administratif est assuré par la D.G.A.L. Pour estimer les risques, il faut prendre en compte le danger représenté par la molécule, mais aussi l'exposition : c'est à partir de ces deux paramètres que l'exercice peut être effectué correctement. Des groupes de travail issus de la Commission participent très activement à cette analyse. L'ensemble des informations relative à la procédure est donné dans la figure 1 extraite d'un document interne de la D.G.A.L.

\section{II — ESTIMATION DES RISQUES}

Le risque est fonction de deux paramètres : la toxicité de la substance en cause et l'exposition.

Une substance très toxique avec une exposition nulle n'entraîne naturellement pas de risque et une substance de toxicité moyenne avec une exposition forte peut être génératrice de dangers majeurs.

Toute la toxicologie se fonde sur ce que l'on appelle la relation " dose/effet", qui s'exprime simplement de la manière suivante :

- il y a proportionnalité entre l'effet toxique et la dose :

- il existe pour tout effet toxique une dose " seuil " audessous de laquelle cet effet toxique ne se manifeste pas.

Au total, on peut donc dire que c'est la dose qui fait le poison.

\subsection{Toxicité}

Il est indispensable de disposer des informations nécessaires à la caractérisation de la toxicité de la substance, toxicité prise au sens large du terme vis-à-vis :

- de l'homme et des mammifères ;

- de l'environnement abiotique (sol et eau) ;

- de l'environnement biotique (oiseaux, milieu aquatique, insectes etc.).

Toutes ces informations ont pour objectif de connaître le profil toxicologique de la substance en cause et d'évaluer ses dangers potentiels. 
Elles doivent permettre de caractériser les relations " dose/effet" sur lesquelles sont fondées les notions de " dose seuil ".

L'élément qui domine l'ensemble est ce qu'on appelle " la dose sans effet " à partir de laquelle on peut tirer des limites de concentrations sous lesquelles les problèmes de sécurité sont réglés.

Cette notion de dose sans effet (D.S.E.) est un concept qui a évolué avec le temps. Il y a quelques années, on se contentait, comme le nom l'indique, de noter les effets observés après administration par différentes voies du produit à des espèces animales diverses. Aujourd'hui, on essaie par des études mécanistiques de comprendre le pourquoi de ces effets afin d'en tirer les meilleurs enseignements pour l'évaluation du risque [1].

Pour le futur, les points suivants évolueront certainement.

\section{I.I Cancérogénicité}

La cancérogénicité reste un point majeur des dossiers à examiner. Dans ce domaine, les études mécanistiques ont apporté quelques nouveautés. La distinction entre génotoxique et non génotoxique est maintenant admise pratiquement par tous et l'estimation des risques se trouve du même coup facilitée. Des outils nouveaux apparaissent comme I'utilisation des animaux transgéniques pour une meilleure connaissance du processus. Notons que la dose maximale tolérée, concept exigé par "I'Environnemental Protection Agency " (EPA - USA) pour les cancérogenèses expérimentales, n'est pas acceptée par les toxicologues de la Communauté.

\section{I.2 Hormonotoxicité}

La toxicité vis-à-vis des glandes endocrines se développe. Elle bénéficie des techniques analytiques qui permettent de doser de manière précise les diverses hormones circulantes. L'impact des toxiques sur ces systèmes complexes sera certainement de plus en plus recherché. En matière de pesticides, certains d'entre eux sont suspectés d'activité œstrogénique affectant ainsi la santé humaine [2].

\subsubsection{Immunotoxicité}

C'est là encore un domaine nouveau de la toxicologie qui apparait. Dans ce cadre, il faut définir une stratégie, utiliser des tests validés et reconnus par tous. Un certain nombre de propositions d'approches sont faites par différents organismes. Ces approches sont toutes fondées sur des étapes à suivre permettant pratiquement de se faire une opinion sur la potentialité immunotoxique des composés examinés. A titre d'exemple, on peut citer la proposition de " European Centre for Ecotoxicology and Toxicology of Chemical " (ECETOC) |3|.

\section{2.l.4 Mélanges}

La toxicité des mélanges est une question, tout au moins au plan théorique, qui se pose systématiquement. Nous évaluons la toxicité des composés pris individuellement. Qu'en est-il de cette toxicité, lorsque nous avons à faire à des mélanges, ce qui est généralement le cas.

A l'heure actuelle, deux types de travaux sont conduits.

Aux Pays-Bas, l'approche est classique, consistant à administrer à des animaux d'expérience des mélanges de plu-

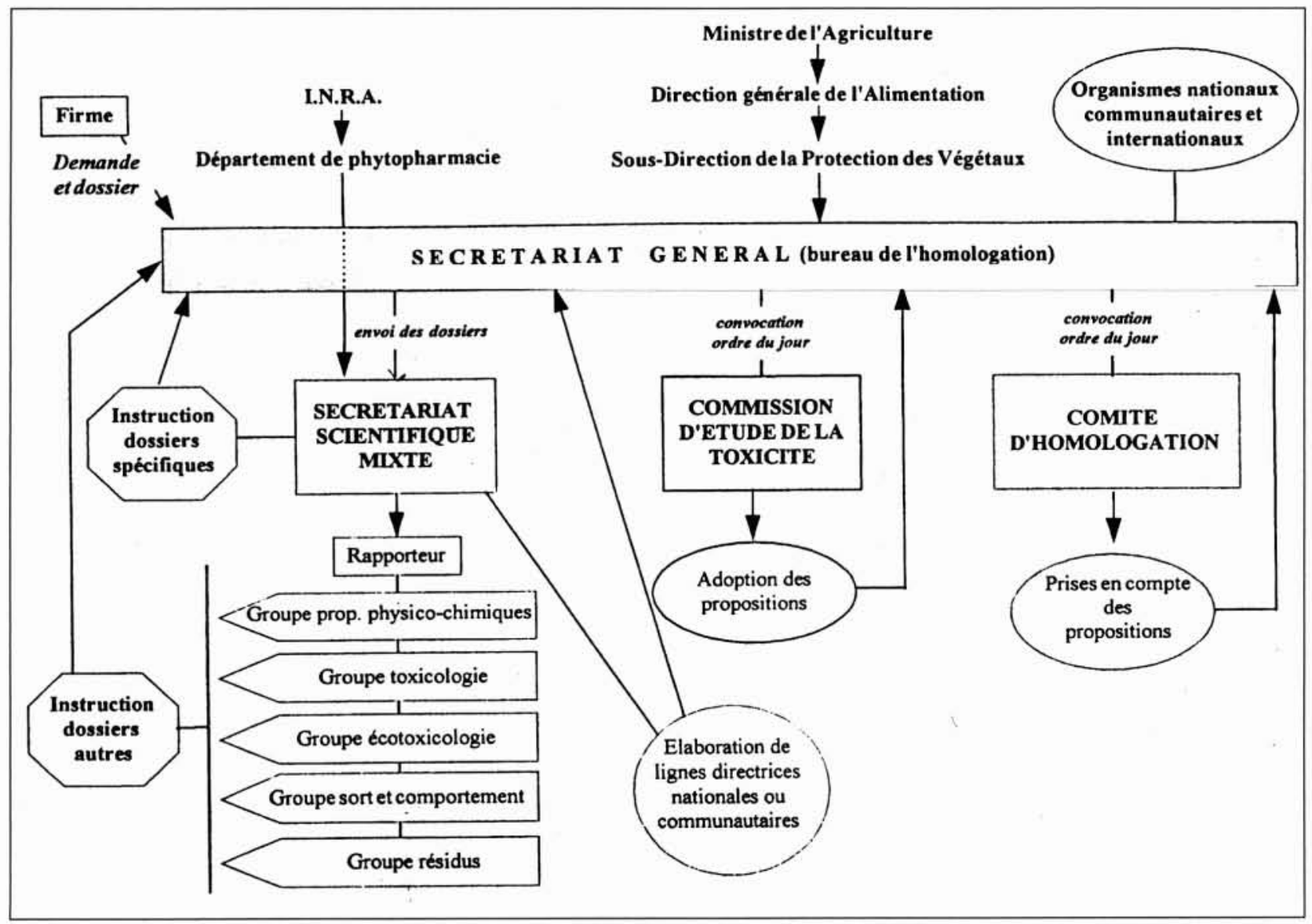

1. Procédure d'homologation des produits phytosanitaires. 
sieurs toxiques à des doses égales à la D.S.E. et des doses plus importantes et de constater les effets. Les premiers résultats montrent qu'au niveau des D.S.E., celles-ci ne sont pratiquement pas modifiées [4].

Aux U.S.A., et plus particulièrement au " Chemical Industry Institut of Toxicology "(C.I.I.T.), l'approche est plus mécanistique. Cependant, cette approche reste surtout valable pour des doses qui sont bien supérieures aux doses sans effet des toxiques testés [5].

Une très bonne analyse des problèmes posés est donnée dans une publication récente [6].

En ce qui me concerne, compte tenu des faibles doses de résidus, je ne pense pas que des effets synergiques puissent être mis en évidence. Ce n'est peut-être pas le cas pour les doses relatives aux manipulateurs et à l'environnement.

\subsection{Exposition}

Il est certain que la mesure de l'exposition réelle (résidus, manipulateur, environnement...) reste un exercice difficile et est sans doute encore mal explorée.

C'est là dessus que devraient porter les efforts les plus importants. La mise en place de laboratoires d'analyses de résidus en France (I.N.R.A. et Délégation Générale de l'Alimentation) est une bonne chose. La mise en route du choix des matières actives, de leurs recherches et de leurs dosages systématiques dans les eaux de surface seront aussi des données intéressantes.

C'est dans ce dernier domaine que les problèmes à résoudre sont sinon les plus complexes, du moins les plus nouveaux, surtout lorsqu'on s'intéresse aux écosystèmes. Dans ce cadre, tous les efforts analytiques qui pourraient être entrepris afin d'harmoniser les méthodes et de mieux connaître l'imprégnation de l'environnement par les produits phytosanitaires sont à retenir. Il faut noter qu'au plan des concepts et en ce qui concerne l'environnement, les notions de concentrations prédictives dans les sols $\left(\mathrm{PEC}_{\mathrm{s}}\right)$, dans l'eau de surface $\left(\mathrm{PEC}_{\mathrm{sw}}\right)$, dans l'eau profonde $\left(\mathrm{PEC}_{\mathrm{gw}}\right)$ et dans l'air $\left(\mathrm{PEC}_{\mathrm{a}}\right)$ et leurs comparaisons avec les concentrations sans effet (No E.C.) sur les espèces vivantes constituent des approches tout à fait dignes d'intérêt.

Cependant, soulignons que ces approches se heurtent à beaucoup de difficultés (validation des modèles, connaissance des No E.C., etc.) et qu'en la matière, un traitement pragmatique au cas par cas, compte tenu de l'évolution des informations et connaissances disponibles, nous parât dans l'état actuel des choses le mieux adapté.

Notons toujours, à propos de l'eau, qu'une approche analytique pour la gestion du risque a été retenue par le Communauté Économique Européenne.

Elle se fonde sur la recherche d'une exposition " nulle " par une mise en œuvre de méthodes de détection de plus en plus sensibles.

C'est sur cette approche qu'ont été édictées les limites CEE pour l'eau potable :
(c) pour un produit
$<0,1 \mu \mathrm{g} / 1$
(c) totale
$<0,5 \mu \mathrm{g} / \mathrm{l}$

Cette approche nous paraît erronée pour les raisons suivantes :

- le " zéro " résidu n'existe pas : il dépend de la sensibilité analytique et le "zéro" analytique devient de plus en plus petit,
- le risque nul n'existe pas.

La philosophie qui a conduit à l'adoption de ces limites s'appuie sur le distinguo que l'on fait entre les xénobiotiques d'origine non naturelle, dont les pesticides font partie, et les xénobiotiques naturels, la volonté affichée étant d'éliminer de l'eau les premiers. Au plan scientifique, cette distinction est pour le moins malheureuse et non fondée. Il est évident que la seule façon d'éliminer les pesticides dans l'eau est d'interdire leur utilisation. Tant qu'ils seront utilisés, il est clair qu'on en trouvera pratiquement toujours par amélioration des techniques analytiques.

Quoi qu'il en soit, la stratégie retenue débouche sur l'illusion d'une fausse sécurité. Elle entraîne des dépenses considérables et des difficultés analytiques majeures, compte tenu des limites de détection envisagées.

Il faut ajouter qu'à ces limites de concentrations $(\mu \mathrm{g} / \mathrm{l}$ ou fractions de $\mu \mathrm{g} / \mathrm{l}$ ) l'eau n'est pas un produit pur. L'expérience montre qu'elle contient une multitude de " contaminants " pratiquement inconnus, ce qui se traduit par un " bruit de fond " très important, dans lequel, en définitive, la présence de quelques produits phytosanitaires aux concentrations envisagées peut apparaître comme mineure.

Il est donc important de faire savoir :

- qu'une norme analytique n'a pas de sens toxicologique et que lorsqu'elle est dépassée : cela n'implique pas un risque toxicologique pour l'homme ou pour l'environnement ;

- qu'une norme analytique doit être considérée comme un objectif de qualité et non pas comme un objectif de salubrité.

Cette distinction entre norme de qualité et norme de salubrité n'est pas facile à expliquer et à faire comprendre à la grande majorité de nos concitoyens. Il n'en demeure pas moins vrai que les autorités sanitaires doivent être à même, lorsque la limite de $0,1 \mu \mathrm{g} / \mathrm{l}$ est dépassée, de faire comprendre et admettre que la santé publique n'est pas forcément menacée et que les dispositions à prendre dépendent de fait des concentrations réellement retrouvées.

Notons qu'une nouvelle directive européenne est en cours d'élaboration. Selon les propositions faites, la valeur de $0,5 \mu \mathrm{g} / \mathrm{l}$ serait abandonnée et celle de $0,1 \mu \mathrm{g} / \mathrm{l}$ maintenue tant que de nouvelles données scientifiques ne permettraient d'aboutir à un consensus sur des limites toxicologiques acceptables. Il y aurait là un net progrès.

En conclusion, à partir des données précédentes, il apparaît que la procédure de l'enregistrement des produits phytosanitaires doit être faite de manière à déboucher sur une utilisation telle que celle-ci conduise à une sécurité d'emploi manifeste, en particulier par tous moyens visant à diminuer l'exposition. Dans ce cadre, on peut définir des doses journalières acceptables (D.J.A.). A partir des D.J.A., il est possible de déduire pour l'eau, selon l'Organisation Mondiale de la Santé, des valeurs guides ou selon la Communauté Européenne des valeurs limites, expressions de nature toxicologique et non pas analytique.

Les décisions devront donc s'appuyer sur le type d'analyse que nous avons développé et être fondées sur des données scientifiques. Mais elles ne peuvent ignorer la pression sociale. Une bonne décision doit d'abord être applicable, mais aussi acceptable pour la Société. De toutes façons, après la prise de décision, il faut aussi en assurer la gestion et au travers de la communication à la faire comprendre. Néanmoins, il ne faut pas oublier que l'analyse toxicologique permet de dépasser le stade des impressions subjec- 
tives face aux problèmes d'exposition à des contaminants chimiques et des produits phytosanitaires en particulier. Le simple fait de déceler la présence d'une substance dans notre environnement n'implique pas nécessairement l'existence d'une menace, voire d'un danger. Il faut reconnaître que la toxicologie est en perpétuelle évolution et qu'il ne lui appartient pas de décider à elle seule des choix de société. Mais dans ce domaine, sa contribution parait devoir être indispensable et pratiquement irremplaçable.

\section{BIBLIOGRAPHIE}

[1] Mc Clellan R. O., 1994. - Application of mechanistic data in toxico$\log y /$ risk assessment. C.I.I.T. Activities - Vol. 14. $\mathrm{N}^{\circ} 11,1-8$.

[2] HILEMAN B., 1994. - Environmental oestrogens linked to reproductive abnormalities, cancer - C \& EN, 14, January 31 1994,19-23 .

[3] ECETOC, 1994. - Immunotoxicity : Hazard identification and risk characterisation - Monography $\mathrm{N}^{\circ} 21$, Septembre 1994, $35 \mathrm{p}$.

[4] Feron V. J. et al, 1993. - Combination toxicology : from challenge to reality. Toxicology Tribune $\mathrm{N}^{\circ} 14,1-3$.

[5] BOND A. J. and MEDINSKi M. A., 1993, - Assessing interactive effects from exposures to chemical mixtures. C.I.I.T. Activities, Vol. $13, \mathrm{~N}^{\circ} 3$ March 1993, 1-3

[6] Mumtaz M.M. et al, 1993. - Risk assessment of chemical mixtures : Biologic and toxicological issues. Fundan Appl. Toxicol. 21, 258-269. 